\title{
Value of D-dimer levels for the diagnosis of pulmonary embolism: An analysis of 32 cases with computed tomography pulmonary angiography
}

\author{
HUI GAO ${ }^{1,2}$, $\mathrm{HU}^{2} \mathrm{LI}^{3}$ and YANJING $\mathrm{LI}^{2}$ \\ ${ }^{1}$ Department of Radiology, The First Affiliated Hospital of Xi'an Jiaotong University, Xi'an, Shaanxi 710061; \\ ${ }^{2}$ Department of Radiology, The Affiliated Hospital of Yan'an University; ${ }^{3}$ Cardiovascular Medicine Center, \\ The Affiliated Hospital of Yan'an University, Yan'an, Shaanxi 716000, P.R. China
}

Received January 16, 2018; Accepted June 6, 2018

DOI: $10.3892 /$ etm.2018.6314

\begin{abstract}
D-dimer levels correlate with the extent of pulmonary embolism (PE) on computed tomography pulmonary angiography (CTPA), but the use of D-dimer alone for screening and diagnosing $\mathrm{PE}$ remains controversial. This study compared the utility of plasma D-dimer levels for diagnosing PE with that of CTPA (gold standard). This was a retrospective analysis of 32 consecutive patients with suspected PE at the Affiliated Hospital of Yan'an University between OctoberC 2010 and March 2011. Blood was sampled before thrombolytic therapy was administered. D-dimer levels were measured using an automatic system. CTPA was used to diagnose PE and was performed within $48 \mathrm{~h}$ of blood sampling using a Siemens Somatom Sensation 64 computed tomography (CT) scanner. ROC curve analysis was performed to evaluate the diagnostic utility of D-dimer levels, with CTPA as the gold standard. Among the 32 patients, CTPA identified 26 patients with PE and 6 patients without obvious abnormality. Using a threshold value of $1.3 \mu \mathrm{g} / \mathrm{ml}$ for D-dimer level, the diagnosis of PE was achieved with a sensitivity of $96.2 \%$, specificity of $50.0 \%$, positive predictive value of $89.3 \%$, negative predictive value of $75.0 \%$ and accuracy of $87.5 \%$. D-dimer levels were significantly higher in patients positive for PE on CTPA than in those negative for PE on CTPA $(9.85 \pm 7.14$ vs. $2.82 \pm 2.65 \mu \mathrm{g} / \mathrm{ml}, \mathrm{P}=0.001)$. Based on ROC curve analysis, the optimal threshold value for D-dimer level in the diagnosis of PE was $1.9 \mu \mathrm{g} / \mathrm{ml}$. To conclude, D-dimer could be a simple, fast and inexpensive screening method for excluding a diagnosis of PE.
\end{abstract}

Correspondence to: Dr Yanjing Li, Department of Radiology, The Affiliated Hospital of Yan'an University, 43 North Street, Baota, Yan'an, Shaanxi 716000, P.R. China

E-mail: 331876652@qq.com

Key words: pulmonary embolism, 64-slice spiral computer tomography, pulmonary angiography, D-dimer

\section{Introduction}

Pulmonary thromboembolism, or pulmonary embolism (PE), is a clinical and pathophysiological syndrome resulting from obstruction of a pulmonary artery or its branches by emboli from the venous system or right heart, causing dysfunction of the pulmonary circulation and respiratory system $(1,2)$. The incidence of PE in the USA and Europe is $60-75$ per 100,000 persons per year (3-6). The incidence of PE is thought to be lower in Asia $(7,8)$, but recent data suggest that the incidence and causes of PE may be similar between Chinese and Caucasian populations (9). Hospitalization and childbirth (i.e., the postpartum period) increase the risk of PE (10-12). Nevertheless, $\mathrm{PE}$ is a dangerous condition (2) that leads to significant right heart dysfunction (13), and the 30-day all-cause mortality is $13 \%$ (14). A major issue in the diagnosis of PE is that some symptoms (such as dyspnea, chest pain, cough and/or fever) are not specific to PE and may be obscured by symptoms caused by another condition $(2,15-17)$.

At present, the main methods used for the diagnosis of $\mathrm{PE}$ are laboratory investigations and imaging. Digital subtraction angiography (DSA) of the pulmonary artery is considered the most accurate (gold standard) method for diagnosing PE (2,15-17). Nevertheless, DSA is an invasive technique that is associated with complications, cannot show emboli in the peripheral branches of a pulmonary artery, and cannot be repeated many times because of its invasiveness. Therefore, DSA is not the first choice method for screening or diagnosing PE $(2,16)$.

Recent developments in computed tomography (CT) have led to multi-slice spiral CT being adopted in clinical practice for the diagnosis of PE, and CT is now favored over DSA (2). Great improvements in temporal resolution and scanning speed have resulted in CT pulmonary angiography (CTPA) now being widely used in the diagnosis of PE (18-23).

$\mathrm{D}$-dimer is a fibrin degradation product generated from blood clot degradation by fibrinolysis (24). Blood D-dimer levels can be used to help diagnose thrombosis (24). Previous studies reported that $\mathrm{D}$-dimer levels in patients with acute $\mathrm{PE}$ are increased significantly (25-27). Nevertheless, some controversies still exist about the use of D-Dimer for diagnosing 
PE, including the optimal threshold value that should be used and issues relating to other causes of elevated D-dimer levels $(28,29)$. Some studies suggested that D-dimer could be used alone for PE screening, avoiding unnecessary CTPA scans $(30,31)$. In addition, D-dimer levels correlate with the extent of PE on CTPA (32). Nevertheless, the use of D-dimer alone is still controversial, and additional studies are still necessary to determine the cut-off point of the D-dimer level that should be used in the diagnosis of PE. Therefore, the aim of the present study was to conduct a comparative analysis of plasma D-dimer levels and CTPA for the diagnosis of PE and to determine the optimal threshold for D-dimer level, using CTPA as the gold standard.

\section{Materials and methods}

Study design and subjects. This was a retrospective analysis of 32 consecutive patients with suspected PE at the Affiliated Hospital of Yan'an University between October 2010 and March 2011. This study was approved by the ethics committee of the Affiliated Hospital of Yan'an University. The need for individual consent was waived by the committee because of the retrospective nature of the study.

The inclusion criteria were: Symptoms of acute chest pain and dyspnea; and a suspected diagnosis of PE. The exclusion criteria were: Past history of PE; anticoagulation therapy received before blood sampling; known allergy to the contrast agent used for CTPA; hepatic or renal dysfunction; unable to cooperate with the examinations; and data required for the analysis were missing from the medical records.

Plasma D-dimer levels. Blood sampling was done before any thrombolytic therapy was administerd. Plasma D-dimer levels were measured using a CA-7000 automatic coagulation instrument (Sysmex Corporation, Kobe, Japan), and the assay was based on the latex agglutination test. The positivity threshold was $1.3 \mu \mathrm{g} / \mathrm{ml}$.

CTPA. CTPA was performed after blood sampling using a Siemens Somatom Sensation 64 CT scanner (Siemens Healthineers, Erlangen, Germany); the interval between the two examinations was less than $48 \mathrm{~h}$. Non-ionic water-soluble iodinated contrast agent (Ultravist $370 \mathrm{mgI} / \mathrm{mg}$ ) was injected at a dose of 1.2-1.5 ml/kg and a rate of 3.5-4.0 ml/s using a Stellant D high pressure injector (Medrad Inc., Warrendake, PA, USA). Each patient was placed in the supine position on the examination bed with both arms above his/her head. Scanning was performed from the bottom of the lungs to the apex of the lungs during a single breath hold. The scanning parameters were: $120 \mathrm{kV}, 85-160 \mathrm{~mA}$, rotation time $0.33 \mathrm{sec}$, pitch 1.0 , and matrix $512 \times 512$. Time-density curves were obtained for all patients.

Image post-processing. Standard reconstruction of the original images was carried out for all included cases. The reconstructed images had a layer thickness of $0.6 \mathrm{~mm}$ and an interval of $0.4 \mathrm{~mm}$. The mediastinal window (window width $350 \mathrm{HU}$, window level $50 \mathrm{HU}$ ), lung window (window width 1,200 $\mathrm{HU}$, window level -500 HU) and PE window (window width 1,000 HU, window level $400 \mathrm{HU}$ ) were reconstructed (33). All data were transmitted to a Leonardo workstation (Siemens Healthineers), and 3D imaging software was used for post-processing, which included multiple planar reformation (MPR), maximum intensity projection (MIP) and volume rendering (VR).

Image analysis. All images were analyzed using a Leonardo workstation (Siemens Healthineers). The axial lung parenchyma, mediastinum, great cardiac vessels, inferior phrenic artery, posterior intercostal artery, bronchial artery and internal thoracic artery were observed by two experienced radiologists, and lesion site, shape, size, number and density were recorded. In the case of discrepancy, consensus was reached through discussion with a third party (an associate chief physician).

Statistical analysis. Continuous data were tested for a normal distribution using the Kolmogorov-Smirnov test. Normally distributed continuous data are presented as means \pm standard deviations. Categorical data are presented as frequencies. Contingency tables were constructed to evaluate the accuracy of using the D-dimer level to diagnose PE, with CTPA as the gold standard. Receiver operating characteristics (ROC) curve analysis was carried out to validate the results. Analyses were carried out using SPSS v.13.0 (SPSS Inc., Chicago, IL, USA). $\mathrm{P}<0.05$ was considered to indicate a statistically significant difference.

\section{Results}

Clinical characteristics of the subjects. The present study included 16 males and 16 females with an average age of $58.5 \pm 16.6$ years (range, 21 to 90 years). The main symptoms were chest pain, with or without cough, shortness of breath and dyspnea. Among the 32 patients, there were six patients with deep vein thrombosis, seven patients with a recent surgical history (four patients with a history of general surgery, two with a history of orthopedic surgery and one with a history of urologic surgery), two patients in the postpartum period, three patients with a history of coronary heart disease, and 12 patients with a history of smoking (Table I). CTPA was used for the diagnosis of PE: Among the 32 patients, there were 26 patients with PE and six patients without obvious abnormality. No patients had an allergic reaction to the contrast agent.

Sites of PE. A total of 576 pulmonary arteries were examined in the 32 patients. As seen in Fig. 1, PE was most commonly observed in the medial, lateral, anterior and posterior basal segmental arteries of the right lung, the anterior, lateral and posterior basal segmental arteries of the left lung, and the dorsal segmental artery of the left lung.

Comparison of D-dimer levels and CTPA results. Table II presents a comparison of the results of $\mathrm{D}$-dimer level measurements (with a positive result defined as a plasma D-dimer level $>1.3 \mu \mathrm{g} / \mathrm{ml}$ ) and CTPA. There was no significant difference in the diagnosis of PE between the D-dimer test and CTPA. Using CTPA as the gold standard, D-dimer had a sensitivity of $96.2 \%$, a specificity of $50.0 \%$, a positive predictive value of $89.3 \%$, a negative predictive value of $75.0 \%$, and an accuracy 
Table I. Characteristics of the 32 patients with suspected PE.

\begin{tabular}{lc}
\hline Characteristics & $\mathrm{n}$ \\
\hline Deep vein thrombosis & $6 / 32$ \\
Recent surgery & $7 / 32$ \\
Postpartum period & $2 / 32$ \\
Trauma & $2 / 32$ \\
Bedridden & $1 / 32$ \\
Chest pain & $5 / 32$ \\
Dyspnea & $1 / 32$ \\
Shortness of breath & $13 / 32$ \\
Hemoptysis & $2 / 32$ \\
Fever & $2 / 32$ \\
Cough and expectoration & $4 / 32$ \\
Incidental finding in abdominal CT scan & $2 / 32$ \\
\hline
\end{tabular}

PE, pulmonary embolism; CT, computed tomography.

of $87.5 \%$ for the diagnosis of PE. Plasma D-dimer level $>1.3 \mu \mathrm{g} / \mathrm{ml}$ was defined as positive.

Comparison of plasma D-dimer levels between patients positive and negative for PE on CTPA. Among the 32 patients, there were four patients with $\mathrm{D}$-dimer levels in the normal range (i.e., $\leq 1.3 \mu \mathrm{g} / \mathrm{ml}$ ) and 28 patients whose D-dimer levels were $>1.3 \mu \mathrm{g} / \mathrm{ml}$. D-dimer levels were significantly higher in patients with a positive CTPA diagnosis of PE than in patients negative for PE on CTPA $(9.85 \pm 7.14$ vs. $2.82 \pm 2.65 \mu \mathrm{g} / \mathrm{ml}$, $\mathrm{P}=0.001$; Table III).

Analysis of the correlation between the maximal cross-sectional area of the obstructed blood vessel and the $D$-dimer level. As shown in Table IV, there was no correlation between plasma D-dimer level and the maximal cross-sectional area of the obstructed blood vessel.

Methodological evaluation of D-dimer concentration test in the diagnosis of PE. Based on the ROC curve analysis (Fig. 2), the best threshold value of D-dimer was $1.9 \mu \mathrm{g} / \mathrm{ml}$ for the diagnosis of PE, with an area under the curve (AUC) of 0.84 . Using this threshold, the expected negative predictive value was the highest, at $75 \%$.

Representative CTPA images. Representative CTPA images are shown in Fig. 3. In the present study, there were four patients without obvious change in the lung parenchyma, two with scar-like changes in the lung tissues, fifteen with pulmonary infarction, one with non-pulmonary infarction exudation, one with mosaic attenuation of the lung parenchyma, four with pleural effusion, and two with pericardial effusion.

\section{Discussion}

D-dimer levels correlate with the extent of PE on CTPA, but the use of D-dimer alone for the screening and diagnosis of $\mathrm{PE}$ is still controversial $(28,29)$. Notably, there is a paucity of data regarding the utility of D-dimer for the diagnosis of PE in the Chinese population, and the optimal threshold value for Chinese individuals has yet to be established. Therefore, this study aimed to conduct a comparative analysis of plasma D-dimer levels and the results of CTPA (gold standard) in a cohort of Chinese patients with suspected PE. Our novel findings revealed that $1.9 \mu \mathrm{g} / \mathrm{ml}$ may be the optimal cut-off value for D-dimer when screening for PE in the Chinese population. Furthermore, the sensitivity of the D-dimer test for PE was very high (approaching 100\%). The results suggest that D-dimer could be a simple, fast and inexpensive method for excluding a diagnosis of PE.

$\mathrm{PE}$ is the third most common acute cardiovascular disease, after myocardial infarction and stroke. Thousands of people die of PE each year because they are not diagnosed and treated in time. If they could be treated in time, the fatality rate could be reduced (34). The incidence of PE is thought to be lower in Asia than in western countries $(7,8)$, but recent data suggest that the incidence and causes of PE are similar between Chinese and Caucasian populations (9). A previous study showed an increase in the incidence of PE from the south to the north of China (11), similar to a trend in the incidence of stroke and myocardial infarction (11). This variation is probably due to differences in the prevalence of hypertension, dyslipidemia, obesity and diabetes between regions (35). In the northern Shaanxi Province of China, the fatality rate and misdiagnosis rate of $\mathrm{PE}$ are much higher than national levels due to cultural and economic constraints. Clinical symptoms and laboratory investigations have low sensitivity and specificity for the diagnosis of PE (2,15-17), and often only the detection of plasma $\mathrm{D}$-dimer levels is used (25-27). Therefore, imaging remains a good method for the diagnosis of PE, but access to imaging systems, costs, radiation exposure and the invasiveness of angiography limit their use in developing countries such as China. In addition, the sensitivity of CTPA is limited by respiratory motion artifact, overweight resulting in low signal-to-noise ratio, pulmonary artery catheterization artifact and artifact caused by uneven mixing of contrast agent in the lumen of the pulmonary artery. The scanning technique also has limitations related to the settings used for window width and position, superior vena cava artifact caused by the beam hardening phenomenon, lung window algorithm artifact and stepladder artifact. Anatomical factors (perivascular lymph nodes, illusion of vascular bifurcation and unfilled pulmonary vein around the pulmonary artery) and pathological factors (mucus plug in the trachea, edema around the walls of blood vessels, localized increase in pulmonary artery resistance, pulmonary artery stump thrombosis, primary pulmonary artery sarcoma and other tumor thrombi) also affect CTPA (21). Therefore, even if CTPA has shown good diagnostic value for PE (18-23), it remains imperfect; hence, more convenient and rapid approaches are needed.

$\mathrm{D}$-dimer is a small dimer produced by plasmin, and the D-dimer content of normal human plasma is very low. A variety of diseases associated with coagulation events can increase D-dimer levels, and the plasma level of D-dimer is increased when thromboembolism occurs (24), such as PE (25-27). Perrier et al (25), reported that D-dimer had $99.5 \%$ sensitivity and $41.0 \%$ specificity for PE when using CT or DSA as the gold standard. A meta-analysis by Crawford et al (26), 

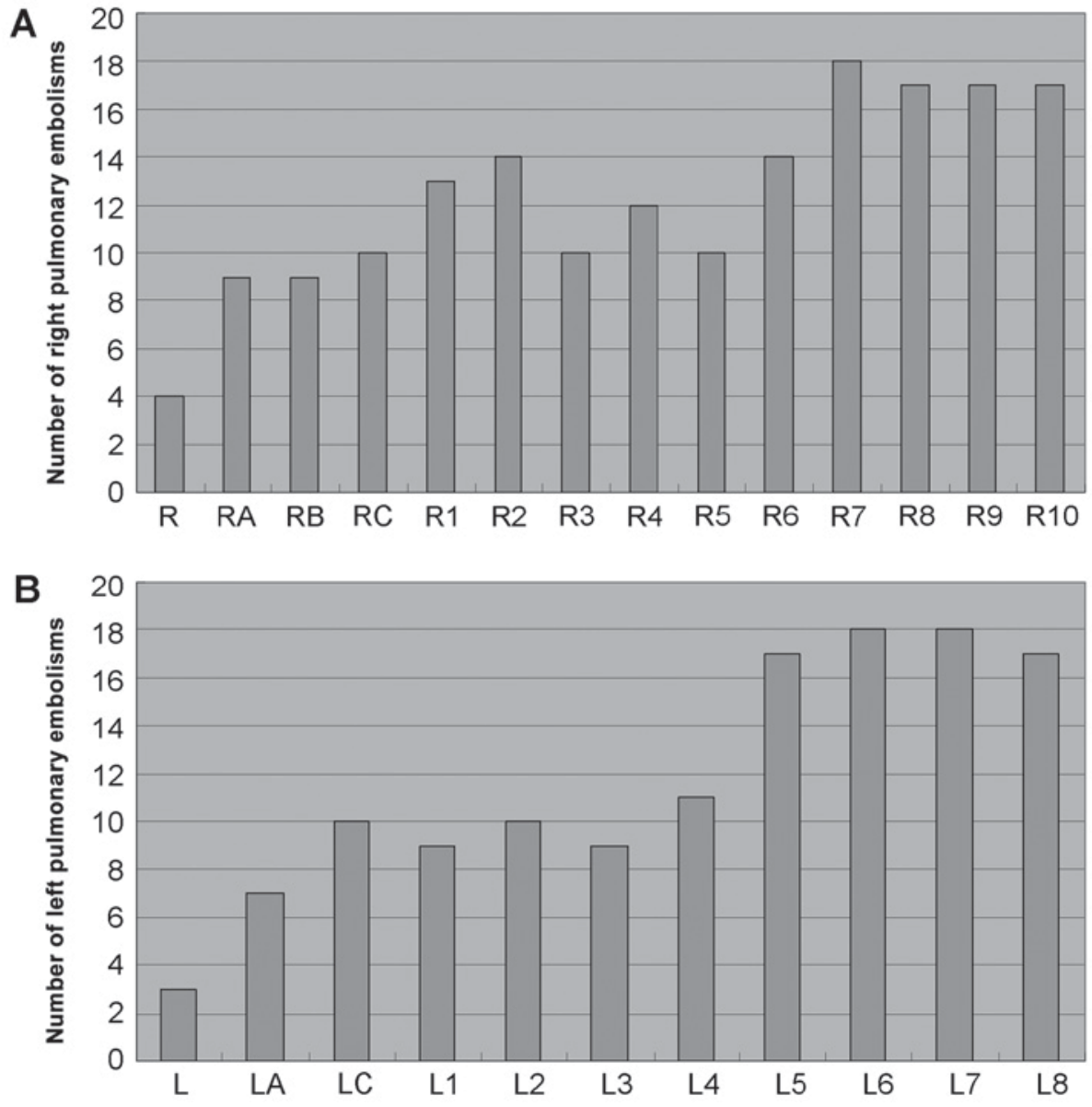

Figure 1. Number of pulmonary embolisms. (A) Right lung. (B) Left lung. R: Right main pulmonary artery; RA: Superior lobar artery of the right lung; RB: Middle lobar artery of the right lung; RC: Inferior lobar artery of the right lung; R1: Apical segmental artery of the superior lobe of the right lung; R2: Anterior segmental artery of the superior lobe of the right lung; R3: Posterior segmental artery of the superior lobe of the right lung; R4: Medial segmental artery of middle lobe of the right lung; R5: Lateral segmental artery of the middle lobe of the right lung; R6: Dorsal artery of the inferior lobe of the right lung; R7: Medial basal segmental artery of the inferior lobe of the right lung; R8: Anterior basal segmental artery of the inferior lobe of the right lung; R9: Lateral basal segmental artery of the inferior lobe of the right lung; R10: Posterior basal segmental artery of the inferior lobe of the right lung; L: Left main pulmonary artery; LA: Superior lobar artery of the left lung; LC: Inferior lobar artery of the left lung; L1: Apicoposterior segmental artery of the left lung; L2: Anterior segmental artery of the left lung; L3: Superior lingular segmental artery of the left lung; L4: Inferior lingular segmental artery of the left lung; L5: Dorsal segmental artery of the left lung; L6: anterior basal segmental artery of the left lung; L7: Lateral basal segmental artery of the left lung; L8: posterior basal segmental artery of the left lung.

showed that the estimated sensitivity of D-dimer for PE ranged between $80-100 \%$, while specificity ranged between $23-63 \%$. In addition, the diagnostic thresholds may vary among different populations of patients (26) and with age $(36,37)$. Schutgens et al (38), found that D-dimer could be used as the preferred screening method for PE, since its high sensitivity meant that negative results could exclude PE. In the present study, using CTPA as the gold standard, D-dimer had $96.2 \%$ sensitivity, $50.0 \%$ specificity and $87.5 \%$ accuracy for the diagnosis of PE. The reported specificity of D-dimer has varied somewhat between published studies, but in general ranged from $23-63 \%$ in studies that used cut-off values of $0.5-1.2 \mu \mathrm{g} / \mathrm{ml}(25,26,30)$. Importantly, the specificity varies with the D-dimer cut-off value chosen; for example, one study reported a specificity of $41 \%$ for a threshold value of $0.5 \mu \mathrm{g} / \mathrm{ml}$ and $93 \%$ for a threshold value of $4 \mu \mathrm{g} / \mathrm{ml}$ (25). Therefore, our finding of a specificity of $50 \%$ for a cut-off value of $1.3 \mu \mathrm{g} / \mathrm{ml}$ is entirely consistent with these previous studies.

The exact D-dimer threshold for the diagnosis of PE is still controversial $(28,29)$. Indeed, as stated above, a meta-analysis showed that the diagnostic thresholds may vary among populations of patients (26). Our center defines high D-dimer levels as $>1.3 \mu \mathrm{g} / \mathrm{ml}$, but the ROC curve analysis suggested that $1.9 \mu \mathrm{g} / \mathrm{ml}$ was the optimal threshold for the diagnosis of $\mathrm{PE}$ in our patient sample. The determination of an optimal threshold value $(1.9 \mu \mathrm{g} / \mathrm{ml})$ that was lower than the average plasma D-dimer level in patients negative for PE on CTPA $(2.82 \pm 2.65 \mu \mathrm{g} / \mathrm{ml})$ reflects the wide range of D-dimer levels measured both in patients positive for PE on CTPA and those negative for PE on CTPA (as is evident from the standard deviations for both groups). For example, although only one of the 26 patients positive for PE on CTPA had a D-dimer level $\leq 1.3 \mu \mathrm{g} / \mathrm{ml}$, three of the six patients $(50 \%)$ negative for PE on CTPA had a D-dimer level $>1.3 \mu \mathrm{g} / \mathrm{ml}$ (Table II). There are, of course, many reasons why patients negative for PE on CTPA might have an elevated D-dimer level, including the presence of other medical conditions (such as deep vein thrombosis, malignancy, sepsis, acute coronary syndrome, acute aortic syndrome, trauma, recent surgery and pregnancy) (39) and the failure of CTPA to detect PE in segmental branches 
Table II. Comparison of D-dimer levels and the diagnostic results of CTPA.

\begin{tabular}{lrrr}
\hline & \multicolumn{3}{c}{ CTPA } \\
\cline { 2 - 4 } D-dimer & + & - & Total \\
\hline+ & 25 & 3 & 28 \\
- & 1 & 3 & 4 \\
Total & 26 & 6 & 32
\end{tabular}

CTPA, computed tomography pulmonary angiography.

Table III. Differences in plasma D-dimer levels between patients with positive and negative CTPA findings.

\begin{tabular}{lrcc}
\hline CTPA & $\mathrm{n}$ & D-dimer $(\mu \mathrm{g} / \mathrm{ml})$ & $\mathrm{P}$ \\
\hline Positive & 26 & $9.85 \pm 7.14$ & 0.001 \\
Negative & 6 & $2.82 \pm 2.65$ & \\
\hline
\end{tabular}

CTPA, computed tomography pulmonary angiography.

Table IV. Analysis of the correlation between the maximal cross-sectional area of the obstructed blood vessel and the plasma D-dimer level.

\begin{tabular}{lccc}
\hline & Mean \pm standard deviation & $\mathrm{r}$ & $\mathrm{P}$ \\
\hline $\mathrm{S}\left(\mathrm{mm}^{2}\right)$ & $1.00 \pm 0.47$ & 0.17 & 0.435 \\
D-dimer $(\mu \mathrm{g} / \mathrm{ml})$ & $10.16 \pm 6.61$ & & \\
\hline
\end{tabular}

S, maximal cross-sectional area of the obstructed blood vessel.

(i.e., a false negative CTPA diagnosis). Notably, deep vein thrombosis, recent surgery, recent pregnancy and a history of coronary heart disease were reported in some of the patients in the present study. ROC curve analysis identifies the optimal threshold based on the Youden index, thus the optimal cut-off point is where the sum of the sensitivity and specificity is maximal. It so happens that this threshold in our cohort is $1.9 \mu \mathrm{g} / \mathrm{ml}$. The existence of numerous causes of elevated D-dimer levels likely underlies the low specificity of D-dimer for PE.

A previous study showed that $\mathrm{D}$-dimer levels correlate with the extent of PE on CTPA (32). In the present study, no correlation was observed between the maximal surface area of the embolized artery and D-dimer level. This discrepancy could be due to the methods used to assess the extent of PE.

CTPA has been proposed as a first-line investigation for the diagnosis of PE. MPR, MIP, VR and other post-processing techniques are widely recognized as a valid alternative to the DSA gold standard (40). The present study found that CTPA had notable advantages in the diagnosis of PE. Indeed, CTPA is able to visually display the number, location, size, shape and

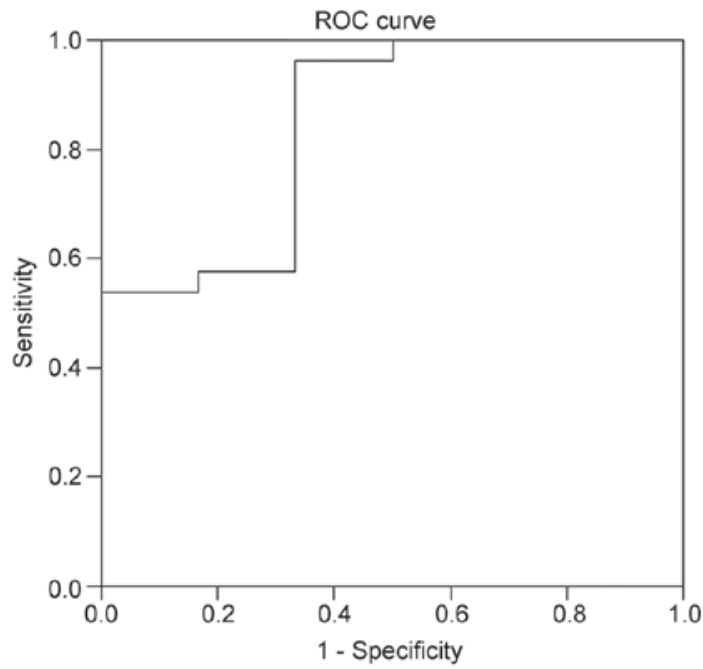

Figure 2. ROC curve analysis of the utility of plasma D-dimer level in the diagnosis of PE. PE, pulmonary embolism.

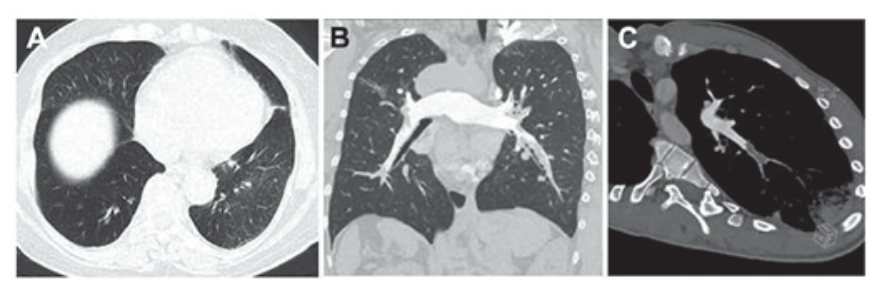

Figure 3. (A) Lung window showing that the field transmittance of the two lungs was uneven. (B) Embolism in the superior segmental artery of the posterior lobe of the right lung; scar-like changes can be seen in the right upper lung. (C) Oblique axis MPR image showing complete embolization of the lateral basal segmental artery of the left lower lung and distal infarcted lung tissue. MPR, multiple planar reformation.

other characteristics of the emboli as well as show changes in the lung tissues after embolization. Moreover, for cases without embolism, it is possible to obtain valuable information about the clinical condition of the lungs and the presence of macroangiopathy, which can explain the clinical symptoms and signs. On the other hand, the detection of D-dimer is convenient, rapid and inexpensive, making it a good PE screening tool (41). Nevertheless, because of its low specificity (26), it cannot be used to make a definitive diagnosis of PE. A previous study had reported that the plasma D-dimer level was closely related to the position and size of the embolism (42). In addition, although CTPA could easily detect emboli in the main pulmonary artery, it could miss emboli in segmental branches, whereas the D-dimer level was elevated in both cases (42). Therefore, we believe that the combination of CTPA and D-dimer can effectively improve the accuracy of $\mathrm{PE}$ diagnosis and reduce the rate of misdiagnosis.

Certainly, there were some limitations. The sample size was small and the number of patients with negative CTPA was small. In addition, because most patients suffered from shortness of breath and dyspnea, ECG-gated CTPA was not performed, and observation of the pulmonary artery near the heart was affected by the heartbeat. Moreover, X-ray exposure during CTPA scanning is an important issue, and future studies should focus on avoiding high radiation doses. Also, 
DSA was not performed to confirm the cases of PE. Finally, the study was retrospective in design, so may have been prone to selection bias and information bias; it also cannot be excluded that unknown confounders influenced our results. However, it should be noted that information regarding D-dimer level and the diagnostic results of CTPA could be reliably obtained from the medical records, hence information bias was likely minimal. Furthermore, a retrospective design is often used a first step in the determination of optimal cut-off values for a biomarker (43). Additional studies are needed to confirm the optimal cut-off value and validate the use of D-dimer levels in the diagnosis of PE in the Chinese population.

In conclusion, measurement of plasma D-dimer level could be a simple, fast and inexpensive method for excluding a diagnosis of PE. Detection of plasma D-dimer levels could avoid unnecessary CTPA examinations and facilitate the early instigation of treatment for $\mathrm{PE}$, thus improving the prognosis of patients.

\section{Acknowledgements}

Not applicable.

\section{Funding}

No funding was received.

\section{Availability of data and materials}

All data generated or analyzed during this study are included in this published article

\section{Authors' contributions}

HG conceived and coordinated the study, designed, performed and analyzed the experiments and wrote the paper. HL and YL performed the data collection, data analysis and revised the paper. All authors reviewed the results and approved the final version of the manuscript.

\section{Ethics approval and consent to participate}

The present study was approved by the ethics committee of the Affiliated Hospital of Yan'an University. All procedures performed in studies involving human participants were in accordance with the ethical standards of the institutional and/or national research committee and with the 1964 Helsinki declaration and its later amendments or comparable ethical standards. The need for individual consent was waived because of the retrospective nature of the study. However, all study participants provided informed consent for the investigations and treatment they received.

\section{Consent for publication}

Not applicable.

\section{Competing interests}

The authors declare that they have no competing interests.

\section{References}

1. Kearon C: Diagnosis of pulmonary embolism. CMAJ 168 : 183-194, 2003.

2. Konstantinides SV, Torbicki A, Agnelli G, Danchin N, Fitzmaurice D, Galiè N, Gibbs JS, Huisman MV, Humbert M, Kucher N, et al: 2014 ESC guidelines on the diagnosis and management of acute pulmonary embolism. Eur Heart J 35: 3033-3069, 3069a-3069k, 2014.

3. Heit JA, Melton LJ III, Lohse CM, Petterson TM, Silverstein MD, Mohr DN and O'Fallon WM: Incidence of venous thromboembolism in hospitalized patients vs. community residents. Mayo Clin Proc 76: 1102-1110, 2001.

4. Huerta C, Johansson S, Wallander MA and García Rodríguez LA: Risk factors and short-term mortality of venous thromboembolism diagnosed in the primary care setting in the United Kingdom. Arch Intern Med 167: 935-943, 2007.

5. Silverstein MD, Heit JA, Mohr DN, Petterson TM, O'Fallon WM and Melton LJ III: Trends in the incidence of deep vein thrombosis and pulmonary embolism: A 25-year population-based study. Arch Intern Med 158: 585-593, 1998.

6. Oger E: Incidence of venous thromboembolism: A community-based study in Western France. EPI-GETBP Study Group. Groupe d'Etude de la Thrombose de Bretagne Occidentale. Thromb Haemost 83: 657-660, 2000.

7. Jang MJ, Bang SM and Oh D: Incidence of venous thromboembolism in Korea: From the health insurance review and assessment service database. J Thromb Haemost 9: 85-91, 2011.

8. Molina JA, Jiang ZG, Heng BH and Ong BK: Venous thromboembolism at the National Healthcare Group, Singapore. Ann Acad Med Singapore 38: 470-478, 2009.

9. Gong JN and Yang YH: Current clinical management status of pulmonary embolism in China. Chin Med J (Engl) 130: 379-381, 2017.

10. Stein PD, Beemath A and Olson RE: Trends in the incidence of pulmonary embolism and deep venous thrombosis in hospitalized patients. Am J Cardiol 95: 1525-1526, 2005.

11. Yang Y, Liang L, Zhai Z, He H, Xie W, Peng X and Wang C; Investigators for National Cooperative Project for Prevention and Treatment of PTE-DVT: Pulmonary embolism incidence and fatality trends in Chinese hospitals from 1997 to 2008: A multicenter registration study. PLoS One 6: e26861, 2011.

12. Tepper NK, Boulet SL, Whiteman MK, Monsour M, Marchbanks PA, Hooper WC and Curtis KM: Postpartum venous thromboembolism: Incidence and risk factors. Obstet Gynecol 123: 987-996, 2014.

13. Shopp JD, Stewart LK, Emmett TW and Kline JA: Findings from 12-lead electrocardiography that predict circulatory shock from pulmonary embolism: Systematic review and meta-analysis. Acad Emerg Med 22: 1127-1137, 2015.

14. Spencer FA, Gore JM, Lessard D, Douketis JD, Emery C and Goldberg RJ: Patient outcomes after deep vein thrombosis and pulmonary embolism: The worcester venous thromboembolism study. Arch Intern Med 168: 425-430, 2008.

15. Jaff MR, McMurtry MS, Archer SL, Cushman M, Goldenberg N, Goldhaber SZ, Jenkins JS, Kline JA, Michaels AD, Thistlethwaite P, et al: Management of massive and submassive pulmonary embolism, iliofemoral deep vein thrombosis, and chronic thromboembolic pulmonary hypertension: A scientific statement from the American Heart Association. Circulation 123: 1788-1830, 2011

16. Goldhaber SZ and Bounameaux H: Pulmonary embolism and deep vein thrombosis. Lancet 379: 1835-1846, 2012.

17. Busse LW and Vourlekis JS: Submassive pulmonary embolism. Crit Care Clin 30: 447-473, 2014.

18. Hayashino $\mathrm{Y}$, Goto $\mathrm{M}$, Noguchi $\mathrm{Y}$ and Fukui $\mathrm{T}$ : Ventilation-perfusion scanning and helical CT in suspected pulmonary embolism: Meta-analysis of diagnostic performance. Radiology 234: 740-748, 2005.

19. Anderson DR, Kahn SR, Rodger MA, Kovacs MJ, Morris T, Hirsch A, Lang E, Stiell I, Kovacs G, Dreyer J, et al: Computed tomographic pulmonary angiography vs. ventilation-perfusion lung scanning in patients with suspected pulmonary embolism: A randomized controlled trial. JAMA 298: 2743-2753, 2007.

20. Quiroz R, Kucher N, Zou KH, Kipfmueller F, Costello P, Goldhaber SZ and Schoepf UJ: Clinical validity of a negative computed tomography scan in patients with suspected pulmonary embolism: A systematic review. JAMA 293: 2012-2017, 2005. 
21. Wittram C, Maher MM, Yoo AJ, Kalra MK, Shepard JA and McLoud TC: CT angiography of pulmonary embolism: Diagnostic criteria and causes of misdiagnosis. Radiographics 24: 1219-1238, 2004.

22. Wittram C, Waltman AC, Shepard JA, Halpern E and Goodman LR: Discordance between CT and angiography in the PIOPED II study. Radiology 244: 883-889, 2007.

23. Stein PD, Fowler SE, Goodman LR, Gottschalk A, Hales CA, Hull RD, Leeper KV Jr, Popovich J Jr, Quinn DA, Sos TA, et al: Multidetector computed tomography for acute pulmonary embolism. N Engl J Med 354: 2317-2327, 2006.

24. Adam SS, Key NS and Greenberg CS: D-dimer antigen: Current concepts and future prospects. Blood 113: 2878-2887, 2009.

25. Perrier A, Desmarais S, Goehring C, de Moerloose P, Morabia A, Unger PF, Slosman D, Junod A and Bounameaux H: D-dimer testing for suspected pulmonary embolism in outpatients. Am J Respir Crit Care Med 156: 492-496, 1997.

26. Crawford F, Andras A, Welch K, Sheares K, Keeling D and Chappell FM: D-dimer test for excluding the diagnosis of pulmonary embolism. Cochrane Database Syst Rev: CD010864, 2016.

27. Le Gal G, Righini M and Wells PS: D-dimer for pulmonary embolism. JAMA 313: 1668-1669, 2015.

28. Pathak VP, Rendon ISH and Mthyala P: Elevated D-dimer is not always pulmonary embolism. Resp Med CME 4: 91-92, 2011

29. Righini M, Van Es J, Den Exter PL, Roy PM, Verschuren F, Ghuysen A, Rutschmann OT, Sanchez O, Jaffrelot M, Trinh-Duc A, et al: Age-adjusted D-dimer cutoff levels to rule out pulmonary embolism: The ADJUST-PE study. JAMA 311: $1117-1124,2014$

30. Eng CW, Wansaicheong G, Goh SK, Earnest A and Sum C: Exclusion of acute pulmonary embolism: Computed tomography pulmonary angiogram or D-dimer? Singapore Med J 50 403-406, 2009.

31. Subramaniam RM, Chou T, Swarbrick M and Karalus N Pulmonary embolism: Accuracy and safety of a negative CT pulmonary angiogram and value of a negative $\mathrm{D}$-dimer assay to exclude CT pulmonary angiogram-detectable pulmonary embolism. Australas Radiol 50: 424-428, 2006.

32. Ji Y, Sun B, Juggessur-Mungur KS, Li Z and Zhang Z: Correlation of D-dimer level with the radiological severity indexes of pulmonary embolism on computed tomography pulmonary angiography. Chin Med J (Engl) 127: 2025-2029, 2014.

33. Storto ML, Di Credico A, Guido F, Larici AR and Bonomo L: Incidental detection of pulmonary emboli on routine MDCT of the chest. AJR Am J Roentgenol 184: 264-267, 2005.
34. Nanchal R, Kumar G, Taneja A, Patel J, Deshmukh A, Tarima S, Jacobs ER and Whittle J; from the Milwaukee Initiative in Critical Care Outcomes Research (MICCOR) Group of Investigators: Pulmonary embolism: The weekend effect. Chest 142: 690-696, 2012.

35. Tsai AW, Cushman M, Rosamond WD, Heckbert SR, Polak JF and Folsom AR: Cardiovascular risk factors and venous thromboembolism incidence: The longitudinal investigation of thromboembolism etiology. Arch Intern Med 162: 1182-1189, 2002.

36. Gupta A, Raja AS, Ip IK and Khorasani R: Assessing 2 D-dimer age-adjustment strategies to optimize computed tomographic use in ED evaluation of pulmonary embolism. Am J Emerg Med 32: 1499-1502, 2014

37. Woller SC, Stevens SM, Adams DM, Evans RS, Lloyd JF, Snow GL, Bledsoe JR, Gay DZ, Patten RM, Aston VT and Elliott CG: Assessment of the safety and efficiency of using an age-adjusted D-dimer threshold to exclude suspected pulmonary embolism. Chest 146: 1444-1451, 2014.

38. Schutgens RE, Ackermark P, Haas FJ, Nieuwenhuis HK, Peltenburg HG, Pijlman AH, Pruijm M, Oltmans R, Kelder JC and Biesma DH: Combination of a normal D-dimer concentration and a non-high pretest clinical probability score is a safe strategy to exclude deep venous thrombosis. Circulation 107: 593-597, 2003.

39. Schutte T, Thijs A and Smulders YM: Never ignore extremely elevated D-dimer levels: They are specific for serious illness. Neth J Med 74: 443-448, 2016.

40. British Thoracic Society Standards of Care Committee Pulmonary Embolism Guideline Development Group: British thoracic society guidelines for the management of suspected acute pulmonary embolism. Thorax 58: 470-483, 2003.

41. Castañer E, Gallardo X, Ballesteros E, Andreu M, Pallardó Y, Mata JM and Riera L: CT diagnosis of chronic pulmonary thromboembolism. Radiographics 29: 31-53, 2009.

42. De Monyé W, Sanson BJ, Mac Gillavry MR, Pattynama PM, Büller HR, van den Berg-Huysmans AA and Huisman MV; ANTELOPE-Study Group: Embolus location affects the sensitivity of a rapid quantitative D-dimer assay in the diagnosis of pulmonary embolism. Am J Respir Crit Care Med 165: 345-348, 2002

43. Pepe MS, Etzioni R, Feng Z, Potter JD, Thompson ML, Thornquist M, Winget $\mathrm{M}$ and Yasui Y: Phases of biomarker development for early detection of cancer. J Natl Cancer Inst 93: 1054-1061,2001 